\title{
LA IMÁGEN DE BRASIL EN BOHEMIA DE LOS SIGLOS XVI-XIX.
}

\author{
Oldřich Kašpar*
}

I.

El interẻs de los checos por la problemática del Nuevo Mundo tiene una historia larga. Sus raíces estân en los primeros afios del siglo XVI. En aquella época, alrededor del afio 1506, el impresor eslovaco instalado en el oeste de Bohemia en la ciudad de Pilsen, edita su famoso Escrito de las nuevas tierras y el Nueno Mundo..., que es una versión libre del escrito de Amerigo Vespucho Mundus Novus, ampliada en algunas infromaciones de las fuentes colombinas. ${ }^{1}$ Esta información - la primera conocida hasta el momento - dedicada a los descubrimientos en el ultramar da inicio a una serie de noticias y relaciones sobre el Nueno mundo que nace en el siglo XVI y continúa hasta nuestros días. De otras fuentes significativas recordemos por ejemplo un capítulo intitulado De las nuevas islas, cómo, por quién y cuándo fueron descubiertas, que forma parte de la Cosmografía checa editada en el affo $1554 .^{2}$ Este breve capítulo por primera vez brindó al lector checo una visión global de los descubrimientos en el ultramar y comienzos de la conquista del continente americano. Entre otros, publica por primera vez en idioma checo partes de la segunda y tercera Relación de Cortês que describe la conquista de Tenochtitlán. ${ }^{3}$

Un capítulo específico en el proceso de divulgación de la información referente al Nuevo Mundo lo representa el fin del siglo XVII y prácticamente las tres cuartas partes del siglo XVIII. Fue en este período que salen para América los miembros de la orden jesuita de la antigua provincia checa. Durante estos años, más de 150 desarrollaron sus actividades en el nuevo continente y sus informes, relaciones, crónicas y cartas conservados hasta nuestra época, constituyen una valiosa fuente histórica y etnográfica. Entre los mås significativos sefralamos por ejemplo la crónica tarahumara de Josef Neumann, un precioso manuscrito iluminado de Ignacio Tirsch, gramática de la 
lengua tarahumara de Matyăs Štefl o el diario de los viajes por la peń́nsula de California de Václav Linck. ${ }^{4}$

Los comienzos del interés cientifico moderno por América Latina están vinculados al nombre de Tadeo Haenke (1761) oriundo de Bohemia del norte, miembro de la expedición de Malaspina, quien vivió la mayor parte de su vida en el sur del continente americano. ${ }^{5}$

Una amplia entrada de informaciones se evidencia en las vísperas del siglo XIX y sus primeras décadas en relación con la divulgación del racionalismo iluminado y estrechamente unida a un fenómeno social denominado "el renacimiento nacional checo". El esfuerzo por elevar el nivel cultural del pueblo que durante siglos anteriores había sido oprimido política, económica y culturalmente por la monarquía multinacional de los Habsburgo, se manifiesta también por un interés intensificado por el fervor internacional, aunque se trate en algunos casos de tierras relativamente alejadas.

Sin embargo, las informaciones sobre el Nuevo Mundo no se tomaban solamente de las versiones nacionales y surgidas en el ambiente checo. En los fondos históricos de las bibliotecas checoslovacas y los archivos se conserva una serie de fuentes interesantes - en muchos casos originales -, que nos permiten estudiar las relaciones entre los países checos y el mundo hispanoamericano y luso-brasileño en las distintas etapas históricas. ${ }^{6}$

El relación con la orientación pro-espanola de una parte de la nobleza de la corte praguense en la segunda mitad del siglo XVI y la primera del siglo XVII, encontramos hoy día junto a títulos aislados también colecciones de libros espanoles, reunidas con esmerado cuidado aquí en Bohemia o traídas parcialmente de España, en forma de regalos, dote, etc. Desde este punto de vista, las bibliotecas más significativas de orientación pro-hispánica eran de las casas Pernestăn, Lobkowicz, Ditrichstein etc. En ellas encontramos a veces obras únicas. ${ }^{7}$ En general podemos decir, que en nuestras bibliotecas están representadas prácticamente todas las fuentes de importancia referentes a los descubrimientos en el ultramar y conquista del continente americano, entre ellas primeras ediciones - López de Gomara, Bernal Díaz del Castillo - así como versiones posteriores en latín y otros idiomas - Colón, Cortés, Vespucho y otros. De este modo, la imágen del Nuevo Mundo en los países checos de los siglos XVI - XIX es relativamente bien conocida, se conocen también las posibilidades que tenían las distintas capas sociales de aquella época de obtener informaciones sobre América, empezando por la alta nobleza, clérigos, burguesía, hasta las clases populares. ${ }^{8}$

Gracias a los estudios realizados en este campo en los últimos años podemos sacar la conclusión de que el Nuevo Mundo se hizo parte integrante de las concepciones geográficas e históricas de una parte considerable de la sociedad checa ya a partir del siglo XVI. ${ }^{9}$ 
II.

Una parte integrante de la información global del Nuevo Mundo la constituyen los datos sobre Brasil. Junto a una serie de fuentes en otros idiomas procedentes del siglo XVI, se considera como la primera y a la vez más significativa fuente checa el manuscrito del ano 1590 - Historia de la navegación hacia América a la cual denominan Brasilia, cuyos autores eran dos hermanos checos Pavel Slovåk y Matej Cyrus. El folleto es de hecho una traducción del famoso escrito del clérigo calvinista borgonés Jean de Léry al checo, partiendo de una versión en latín. La traducción no es totalmente fiel en cuanto al contenído, los autores introdujeron algunas variaciones sustanciales. Sobre todo, eliminaron el prefacio y algunas partes polémicas referentes a André Thévet. ${ }^{10}$ La característica de la versión checa la constituyen las notas marginales del manuscrito, que habían sido hechas posteriormente por un lector - evidentemente jesuita - y que documentan su posición intransigente hacia los creyentes no-católicos.

Debido a unas invectivas relativamente fuertes contra la alta nobleza, la traducción manuscrita del escrito de Léry no salió nunca editada en su época, sino fue publicada en el año 1957 por Quido Hodura y Bohuslav Horák. Sin embargo, gracias a los estudios de los inventarios y herencias praguenses ya hoy día está comprobado, que el escrito circulaba en el siglo XVII en el medio burgués en forma de copias manuscritas. ${ }^{11}$

Informaciones aisladas sobre Brasil aparecen en dos compendios históricos cronológicos del tipo humanista, de finales del siglo XVI. En primer lugar es el Almanaque histórico del destacado humanista checo Daniel Adam de Veleslavín, ${ }^{12}$ segundo, se trata del Indice histórico de Benjamin Petrek de Polkovice. ${ }^{13}$

La siguiente onda de informaciones sobre Brasil aparece a finales del siglo XVII y principios del XVIII, aunque ya en el primer tercio del siglo XVII encontramos a los primeros dos imigrantes checos en Brasil. Se trata de emigrantes no-católicos de la época posterior a la batalla de la Montaña Blanca tan fatal para la nación checa, que vinieron a las costas de Brasil a servicio de los holandeses. El primero era el doctor Simon Kohout de Lichtenfeld, el segundo, el capitán Jiřr Kryštof Kaplír de Sulevice. El doctor Kohout murió poco después de su arribo a Brasil, el capitán "George Caplier" cayó en la segunda batalla de Guararapes en el afio $1647 .{ }^{14} \mathrm{Ningu}-$ no de los dos dejó documentos escritos de su estancia y quedan incluso pocos materiales de los jesuitas, ya que los jesuitas de la provincia checa en su mayorfa desarrollaban sus actividades en otras regiones de América Latina. De los que trabajaban directamente en el territorio del actual Brasil, no se 
conservan informaciones o, como en el saso de al parecer primer jesuita de la provincia checa en el continente americano Valentin Stansel, se dedicaban a otros asuntos específicos. ${ }^{15}$

Así, la siguiente ola de entrada de información sobre Brasil esta relacionada con el desarrollo del renacimiento nacional checo, por motivos que en el nivel general fueron expuestos ya anteriormente y a los cuales se une el motivo específico de Brasil. ${ }^{16}$

En la primera y segunda fases del renacimiento nacional checo jugaron un papel importante, no solamente desde el punto de vista de la divulgación de informaciones sobre América Latina, dos destacados representantes de la cultura checa de aquella época, los Kramerius - padre e hijo. ${ }^{17}$ El segundo, Václav Rodomil Kramerius es a la vez autor del segundo trabajo más completo sobre Brasil, editado en este período - Emigrantes a Brasil, ${ }^{18}$ un cuadro más bien novelístico basado en el original alemán de Amélia Schoppe. Una historia romántica de la familia de un emigrante que se desarrolla sobre un fondo exótico para el europeo, la naturaleza brasileña. El libro se hizo muy popular sobre todo en las capas populares, según demuestra su segunda edición.

Brasil aparece con mucha frecuencia en las páginas de las revistas nacionales de los afios treinta y cuarenta del siglo pasado, en forma de breves relatos de viajes, descripciones de las curiosidades naturales y sociales, acontecimientos así como cartas de los emigrantes a sus familiares. La situación de Brasil se aprovechaba a menudo como un instrumento para criticar la situación nacional. Por ejemplo, A. Sabinský, en su relato del viaje a Brasil en la revista Česká v̌cela en el año 1836 critica sobre un ejemplo de Brasil con mucha fuerza a los renegados checos. Al hablar en un momento del relato sobre su acompanante, que exclama: "Soy eu o filio Brazil:"19 [la frase esta mutilada], el autor añadió con un suspiro:

"Me recordé de mi patria, sus montes, ríos, bosques y jardines y se me salieron lágrimas. Hijos compatriotas, me comprenden por la emoción? Mi Antonio era un brasilefio fiel!"’20

Otra reminiscencia aparece en la revista Květy ceské del afno 1834. En el artículo intitulado "Cartas de Brasil" se publica una carta enviada por B. František Bernhaus al hijo. En la introducción se señala con orgullo, que Bernhaus fue a Brasil como especialista de una companía inglesa-brasilefia, "para iniciar y dirigir la extracción de los minerales según lo usual aquí."21 
Una conclusión altamente moralista, que toca la misma cuerda que en ambos ejemplos anteriores, se nota en el artículo "Los extranjeros en Brasil". ${ }^{22}$ El articulo critica severamente la emigración a Brasil, describe la nueva vida de los emigrantes en los colores oscuros y termina con un llamamiento patriótico:

" ¿Cuál sería el final de este canto? Cada cual quedese en su Patria y búsquese un trabajo digno!'23

Igualmente se publican artículos sobre la esclavitud, que se caracterizan por las simpatías hacia la población negra de América y un estricto rechazo de la esclavitud. ${ }^{24}$ También otros pequefios trabajos son interesantes por sus caracteristicas de las diferencias en la mentalidad de la población de los dos países, como p.ej. el artículo "En Río de Janeiro el nivel del trabajo del apuntador en los teatros es superior al nuestro", y otros.

Al final de nuestro breve estudio acerca de la historia de las relaciones checo-brasileñas segin se evidenciaba en el pasado sobre todo por medio de los documentos históricos de la literatura, debemos sefralar, que no era nuestra intención brindar una imagen que pudiera agotar todos los aspectos. No nos sería posible ya que prácticamente desconocemos la realidad vista por la otra parte. Es decir, cómo se reflejaba la existencia de los países checos - Bohemia, Moravia y Silesia en el sentido histórico - en la conciencia de la población de Brasil. Más bien hemos querido hacer um paso más en la presentación de los resultados del estudio de las relaciones entre los países checos y Brasil a travês de la historia en nuestro país. ${ }^{25}$

\section{NOTAS:}

${ }^{1} \mathrm{El}$ facsímile junto con la nueva edición fue publicado en el año 1981 por Pravoslav Kneidl. El facsímile está complementado por una traduccipon del texto al español y por versiones española, inglesa y rusa de los comentarios y notas, que facilitan el trabajo con él a los especialistas extranjeros.

${ }^{2}$ Compare más detalladamente, Oldrích Kašpar, "La Cosmografía checa en el contexto de los trabajos cosmogråficos europeos del siglo XVI", Ibero-Americana Pragensia - en lo adelante sólo IAP/, XI/1977, pp.179-184.

${ }^{3} \mathrm{~A}$ la vez fueron descubiertas en las bibliotecas históricas checoslovacas numerosas ediciones en otros idiomas. Entre las más intersantes está la primera edición en latín del afio 1524, editada en Nuremberg, y la edición mexicana publicada por el arzobispo Francisco Antonio Lorenzana en México en el año 1770. 
${ }^{4}$ Compare Josef Polišenský - Josef Opatrný, Wenceslao Linck y su "Diario del Viaje hacia el Norte de la Península de California", IAP, VI/1972,pp. 173-185; Bohumír Roedl, Kronika Josefa Neumanna o povstant Tarahumari (Crónica de José Neumann sobre el levantamiento de los tarahumaras), Praga 1972, tesis de diploma.

${ }^{5}$ Véase Josef Polišenský, La obra Americanista de Tadeo Haenke y su "Memoria sobre los Ríos Navegables", IAP, VI/1970, pp.199-209; Josef Haubelt, Haenke, Born y Banks, IAP, IV/1970, pp. 179-199. El último resúmen de la problemática haenkiana en la bibliografía nacional aparece en el trabajo de Josef Polišenský, "Tadedš Haenke a krize španělské koloniálint soustavy". ("Tadeo Haenke y la crisis del sistema colonial español"), Sbornik Národn'tho muzea, serie C, tomo XXV/1980, nro. 34, pp. 49-76.

${ }^{6}$ Algunos fueron reflejados en forma de registros de los fondos históricos de orientación hispanoamericana y lusobrasileña. Vésse p.ej. Oldřich Kašpar, Soupis španěls$k y ̉ c h$ tiskú bývalé zamecké knihovny y Roudnici nad Labem nynt deponovaných ve Státnt knihovně ČSR v Praze (Registro de los impresos españoles de la antigua biblioteca del castillo de Roudnice nad Labem, actualmente depositados en la Biblioteca Estatal de la RSCheca de Praga), Praga, SK ČSR, 1984; el mismo autor, Soupis španělskỷch a portugalských tiskú bývalé pražské lobkovické knihovny nynt deponovanỷch ve Státnt knihovně ČSR v Praze (Registro de los impresos españoles y portugueses de la antigua biblioteca de los Lobkwicz, actualmente depositados en la Biblioteca Estatal de la RSCh de Praga), Praga, SK ČSR, 1984.

${ }^{7}$ P.ej. la primera edición de la obra inmortal de Cervantes, Don Quijote, el Manual de conversación en náhuatl de Arenas, editado en México en el año 1611, etc.

${ }^{8}$ Compare Oldrich Kasper - Petr Voit, "Literatura sobre el nuevo mundo en las bibliotecas de Bohemia y Moravia en los siglos XVI y XVII", IAP, XIV/1980.

${ }^{9}$ Véase Oldřich Kašpar, Nový svět v české a evropské literature XVI -XIX. stolett (El Nuevo Mundo en la literatura checa y europea de los siglos XVI-XIX), Praga, 1983.

${ }^{10}$ Compare Oldřich Kašpar, "Actividades francesas en Brasil a mediados del siglo XVI y su repercusión en Bohemia", Cahiers du Monde hispanique et luso-brésilien, 43/1984, pp.35-42.

11 Véase Oldřich Kašpar - Petr Voit, obra citada.

12 Praga $1570,1599$.

13 Praga 1596.

${ }^{14}$ Compare, Nicolette Moutt, "Os Primeiros Tchecos No Brasil", IAP, III/1969, pp.219-224.

${ }^{15}$ Stansel se dedicó a las observaciones astronómicas en la Bahía brasilefra, cuyos resultados publicó en Praga.

${ }^{16}$ Sobre todo representado por la princesa austriaca en el trono brasileño.

${ }^{17}$ Compare Old̆rick Kašpar, "Latinská Amerila v dile V.M. a V.R. Krameriu”" ("América Latina en las obras de V.M. y V.R. Kramerius"), Český lid, aro. 3/1983, pp.162-164.

${ }^{18}$ Václav Rodomil Kramerius, Vystěhovanci do Brazilie aneb chaloupka u Gititonhonhy (Emigrantes a Brasil o La casa junto a Gigitonhonha", Jindrichúv Hradec 1830 . 
19 A Sabinský, "Obrazy zámoŕské I" ("Cuadros del ultramar I"), Česká včela 1836 , nro. 6, p.45.

20 Idem.

21 "Listy z Brazulie" ("Cartas de Brasil"), Květy české, 1834, nro. 36, pp. 295-297.

22 "Cizinci v Brazriii" ("Los extranjeros en Brasil"), Kvĕty české, 1834, nro. 40, pp. $335-336$.

23 Idem, p.336.

${ }^{24}$ P.ej. "Otroci v Jižní Americe" ("Los esclavos en América del Sur"), Květy české, 1834, nro. 15, p.124, "Negerští otroci v Rio de Janeiro" ("Esclavos negros en Rio de Janeiro"), Čskă včela 1835, nro.4749, pp.370 y sgtes.

25 Compare Oldrich Kašpar, "O Interesse dos Tchecos pelo Brasil na Êpoca da Renascença Nacional”, IAP, XII/1978, pp.107-117.

*Centro de Estudios Ibero-Americanos

Univerzita Karlova

Celetina 20, Praha 1

Checoslovaquia 